\title{
PARAMETRIC SURFACES FOR AUGMENTED ARCHITECTURE REPRESENTATION
}

\author{
M. Russo ${ }^{1}$, S. Menconero ${ }^{1}$, L. Baglioni ${ }^{1}$ \\ ${ }^{1}$ Department of History, Representation and Restoration of Architecture, Sapienza University of Rome, Rome, Italy, (m.russo, \\ sofia.menconero, leonardo.baglioni)@uniroma1.it
}

\section{Commission II}

KEY WORDS: 3D modeling, Parametric surfaces, Normal distribution, Complex architecture, Augmented Reality

\begin{abstract}
:
Augmented Reality (AR) represents a growing communication channel, responding to the need to expand reality with additional information, offering easy and engaging access to digital data. AR for architectural representation allows a simple interaction with $3 \mathrm{D}$ models, facilitating spatial understanding of complex volumes and topological relationships between parts, overcoming some limitations related to Virtual Reality. In the last decade different developments in the pipeline process have seen a significant advancement in technological and algorithmic aspects, paying less attention to 3D modeling generation. For this, the article explores the construction of basic geometries for 3D model's generation, highlighting the relationship between geometry and topology, basic for a consistent normal distribution. Moreover, a critical evaluation about corrective paths of existing 3D models is presented, analysing a complex architectural case study, the virtual model of Villa del Verginese, an emblematic example for topological emerged problems. The final aim of the paper is to refocus attention on 3D model construction, suggesting some "good practices" useful for preventing, minimizing or correcting topological problems, extending the accessibility of AR to people engaged in architectural representation.
\end{abstract}

\section{INTRODUCTION}

The growing demand for increasing knowledge about environments is leading to a deep transformation on data transmission. Within this technological and cultural revolution, Augmented Reality (AR) plays an important role. This method applied to architecture allows to associate multilevel descriptive information with representations of any artefact or environment, enlarging the knowledge that user can get from the existing simple observation. It allows connection and interaction with all the multimedia communication tools, from images to videos, from texts to 3D models.

The growing interest in augmented reality is supported on the one hand by a growth in applications and services for data management, on the other hand by the increasing use of performing digital cameras and effective algorithms for marker and markerless recognition (Rabbi and Ulla, 2013). In the last decade the disparity of attention for hardware and software solutions (Van Krevelen and Poelman, 2010) respect to reconstruction methodologies for 3D augmented contents, represents one of the main aspects of weakness of Augmented Architecture. The creation of correct virtual models for AR requires some technical steps, as the generation of polygonal surfaces without topological problems, optimized for visualization without losing data useful for its reading and interpretation. Within the topological problems, the normal direction and distribution plays a fundamental role, defining the level of correct visualization of models and textures.

Usually the normal direction problem doesn't concern output coming from range-based or image-based survey operations, since the models are built according to meshing procedures which considers normal information distribution of the point cloud, evaluated respect to the acquisition reference system. Besides, also the generation of simple solid or surface parametric models (Baglioni, 2008) and native polygonal models requires less control because normal directionality is often correctly calculated in relation with the global model shape. The problem, both geometrical and methodological, arises for all those

${ }^{1}$ In this contribution authors are responsible for the following paragraphs: Introduction, Related works, Pipeline and geometrical issues by M.R.; Geometric analysis by L.B.; complex reconstructive 3D models based on mathematical open surfaces, for which is often required to face a long manually normal correction to obtain a consistent normal orientation, reaching an accurate AR definition (Barlozzini et al, 2018). This kind of surfaces, often used to create $3 \mathrm{D}$ light reconstructive models from complex reality-based ones, involves a major waste of time, affecting the whole modeling process in relation of the level of surface complexity, going to uniform the distribution of the normals direction unless compromising AR representation.

The aim of the research ${ }^{1}$ is to analyse the possible causes that lead to this uneven normal distribution in $3 \mathrm{D}$ complex reconstructive architectonic models composed by several mathematical surfaces. In particular, the purpose is suggesting some methodological behaviour in relation with modelling approach, preventing or minimizing the normal inversion during the construction phase of primitive graphics.

The experimental phase starts from a geometric analysis of the surfaces typology, investigating a possible relationship between the surfaces, their shape, the mathematical nature of their generators or directions, defining at the end a correlation abacus between the types of shapes, construction methods and relative obtained results. At the end a case study is presented, Villa del Verginese (Russo, 2018), a complex building example which requires a necessary awareness about the surface tessellation devoted to an Augmented Reality visualization. The final aim of the article is therefore to analyse this topic, suggesting some general "good practices" both in geometry construction and modification, in order to obtain consistent normal distribution, focusing the attention on 3D content creation for Architecture $\mathrm{AR}$, meanwhile encouraging its use even by the less experienced user.

\section{RELATED WORKS}

AR origin is referred to the early decades of the 20th century with the Memex system, while the first example of AR viewer is attributed to Sutherland in 1965 (Sutherland, 1968), a head mounted display for visualizing a wireframe environment

Parametric model correction for AR by S.M. The conclusion refers to all authors' evaluations, a choral work with convergent opinions. 
correlated to the viewer. The term "Augmented Reality" was first coined by Caudell in the 1990s, indicating a system to facilitate the assembly and maintenance of Boing aircraft. A second definition was provided by Milgram and Kishino (Milgram and Kishino, 1994), conceiving AR as the set of circumstances in which a real environment is "augmented" by virtual objects, through a continuum between the real context and the virtual environment. A first systematic study on augmented reality experiences was concluded only at the end of the 90's (Azuma, 1997), providing a wide and recognized definition of AR, identifying it as a combination of real and virtual environment with 3D data recorded in real time (Azuma et al., 1999; Billinghurst et al. 2001).

In the last twenty years the technological evolution of sensors and management platforms of multimedia content has allowed great advances in the development and versatility of AR, applied in multiple fields (Carmigniani et al., 2011): medicine, defence, architecture, museography or advertising and educational technologies. This latter has highlighted the effectiveness of AR as a dynamic and interactive tool for knowledge (Arduini, 2012; Wu et al., 2013), as shown by the most recent developments in learning with technology (Panciroli and Macauda, 2018), reinforcing the importance of its application on a cognitive level. The possibility of being able to learn through an active and interactive system, characterized by the generic system "learning by doing", allows to open new interesting paths in the field of teaching applied on $\mathrm{CH}$ field, in which Architecture plays a principal role, analysing complex elements in real time through different learning platforms as serious game (Mortara et al., 2014). The enhancement of Cultural Heritage, framed in educational context, focuses on the relationship between Augmented Reality and heritage education (Smith, 2016). For this AR, acting mainly in the visual dimension, opens the possibility of a further evolution in the processes of mediation of Cultural Heritage from the historical, artistic and environmental point of view (Bonacini, 2011). It allows the coexistence of digital and real objects in a new space that does not replace the physical world, but overlaps through a process of digital addition, in synchronicity and interactive way, in order to provide an experience of high content, which reaches increasing levels of concreteness.

AR's first experiments in Cultural Heritage date back to the early 2000 s, proposing pathways of knowledge in architectural or archaeological contexts integrated with reconstructive 3D models (Vlahaki et al., 2001), suggesting applications devoted to CH visualization (Choudary et al., 2009) or analysing its model of communication and acceptance (TAM) by users (Haugstvedt and Krogstie, 2012; Dieck and Jung., 2018). Augmented Reality can be considered today as a tool for democratizing access to digital content, with a communicative and cognitive capacity that favours the ability to understand a complex artefact, from museum contents (Frontoni et al., 2016) to Architecture and Archaeology (Younes et al., 2017), through effective and immediate representations. The AR application to improve $\mathrm{CH}$ knowledge leads to an immediate response in terms of communication and promotion both on an architectural and territorial scale of single static elements (De Paolis, 2012; Murru et al., 2013) or the enhancement of particular monuments condition during time thank AR simulation of past construction and moving interactive events (Empler, 2017).

The background construction of correct 3D models represents one of the main issues for each virtual content representation, a sensible element to consider in 3D architecture modeling field. In fact, the world of architectural models presents a great differentiation inside their typology, passing from small rooms without specification of lighting, materials, furniture to complex geometries characterized by overlaid properties (Sdegno, 2018).
The creation of 3D models of complex and large environments in $\mathrm{CH}$ is still challenging, employing automatic, semiautomatic, and manual techniques as well as active and passive survey approaches (Van Krevelen and Poelman, 2010; Guidi et al., 2014; Luhmann et al., 2014; Remondino et al., 2014). The definition of optimized and correct 3D complex architecture must face different problems, starting from topological and geometrical aspects to $3 \mathrm{D}$ surface processing, in order to satisfy the conversion procedure and the final realistic or effective aim of the model. Within the process of surface construction, an important aspect for the correct model visualization is closely related to the normal surface distribution, which mostly affect the Real-Time Rendering (RTR) visualization. This issue doesn't represent a novelty both in Computer Graphics and Computer Vision field, in which since two decade methodologies and algorithms to control and improve the 3D model visualization in virtual environment have yet been presented (Coorg and Teller, 1997; Borodin et al., 2004), in relation with real-time application for video-games (Ebery, 2006), $\mathrm{CH}$ virtual environment (Potenziani et al., 2015), medicine (Montani et al., 2006) and so forth.

Even if the normal distribution has been well studied both from the mathematical, computational and algorithmic point of view, proposing different corrective tools and strategy, it appears quite difficult to access by the architects mostly involved in the $\mathrm{CH}$ modeling and representation field. On the contrary, they have the possibility to understand and manage the process from a geometric point of view, analysing in deep the different steps of primitive creation and surface generation. Unfortunately, a lot of references focus their attention on several technical aspects related to the complex AR pipeline, but very few of them are devoted to 3D geometric content creation for RTR. This lack leads to entrust individuals' ability to solve the modeling problems in relation to the level of complexity of the model to be represented and the personal skill in model management. For this reason, the article intends to stress the geometrical aspect of modeling generation, in relation with simple and complex primitives, in order to create 3D architectural models for Augmented Reality visualization. The process of construction, analysis and correction of existing models will be addressed from a geometric point of view, integrating this experimental activity and data results with the consistent background on the topic.

\section{PIPELINE AND GEOMETRICAL ISSUES}

The definition of 3D models for RTR in AR requires the knowledge of some well-defined passages and relative bottlenecks. It should be appropriate in such sense to start from the existing relationship between the real world, in our case Architecture, Augmented Reality and virtual models, recalling Miligram and Kishino's model "Reality-virtuality continuum". In this schema (Fig. 01) a first interconnection that have a direct relation with the reconstructed geometric models can be established.

Virtual Continuum (VC)

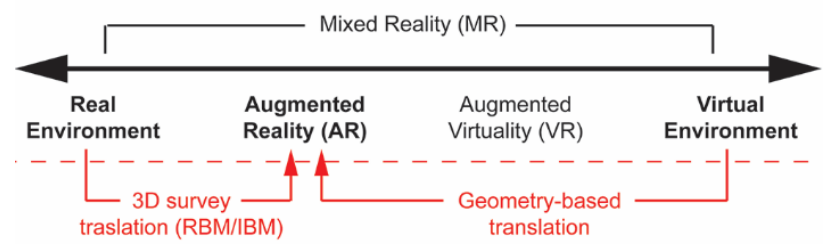

Figure 1: Miligram and Kishino's schema integrated with two different translation paths towards AR output referred to architecture field. 


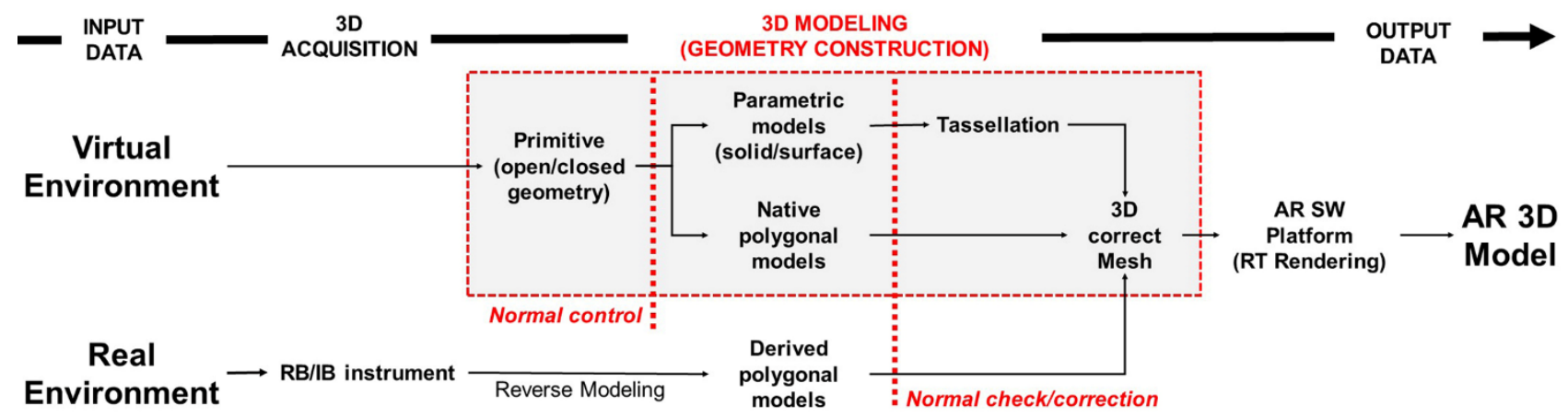

Figure 2: Pipeline from Virtual/Real Environment to AR 3D architectural model definition, with detail on 3D model construction.

In fact, according to the principles of direct relationship between scale, proportions, proximity factors and point of view, there is an evident link between the real world and Augmented Reality, mediated by tracked sensors which enrich environment data. Compared to the original schema, even digital survey methodologies, based on active and passive sensors, can assume the role of "medium" from real to digital world, defining virtual models which are suitable from the topological point of view for RT representation in AR (Fig. 01).

Besides, the connection between virtual environment and its visualization in $\mathrm{AR}$ is quite different. Considering the direct passage between these two worlds, it is strongly conditioned by the nature of the geometric model and its generative primitives. For this reason, an addition schema (Fig. 2) devoted to highlight the architectural 3D model construction in the whole AR pipeline has been suggested, in order to analyse the geometrical aspects. The schema starts again from the real and virtual environment, this time not opposed semantically but considered in parallel. In the last decades the whole showed pipeline has seen a remarkable development, principally due to technological and algorithmic advances. From one side this has led to focus the attention both on 3D acquisition instruments and visualization systems devoted to AR. On the other hand, the introduction of sophisticated algorithms for 3D RTR visualization combined with increasing computational HW and SW capabilities has allowed a significant advance in 3D data management and visualization inside Augmented Reality platforms. As a direct consequence, this has led to not deepen the aspect and bottlenecks related to the construction of the 3D model, quite important in the reverse modeling process but fundamental for the generation of native virtual models, leaving to the personal ability to manage the whole process in relation with the modelling platform. For this reason, the article focuses on this aspect, highlighted in dashed red line in Figure 2, trying to retrace some of the most common problems that can occur in the 3D geometric model construction.

Within this aspect, well known in the Computer Graphics and Computer Vision field, the research will deal with 3D geometric construction, deepening the distribution of the normal surfaces' topic. This represents one of the main bottlenecks of the entire 3D modelling and visualization process, requiring both a prior management and a posteriori verification, in order to avoid misunderstanding in AR visualization.

Returning to the general schema (Fig. 2), the application of range-based (RB) or image-based (IB) techniques framed in the reverse modeling process lead to create reality-based polygonal models, derived from the acquired data, which can be introduced within the AR visualization process, enriching the $3 \mathrm{D}$ existing libraries. The editing activities carried on in the reverse modeling process foresee the correction of existing topological mesh errors, making a verification of the normal distributions for $A R$ visualization irrelevant (Fig. 02).
Starting from the Virtual Environment, it becomes essential to analyse which kind of generative primitives and complex geometries define virtual 3D models, reminding the well-known division in Computer Graphics between solid modeling, parametric and polygonal surfaces. The first two families, although involving different construction methods, both require the further tessellation passage, which allows to transform them into polygonal models useful for RTR in AR.

Of course, this transformation is irrelevant when working directly with polygonal surfaces. As will be detailed later, this transformation from mathematical model to a numerical one (mesh) does not change the topological surface characteristics including the normal distribution, which preserves the set-up defined during the geometric construction.

Therefore, within the construction process of 3D models, it would be appropriate to provide for a normal distribution management during surfaces generation and a final check, this latter aimed at controlling the final output correcting any additional topological anomalies present in the 3D model. This final analysis can be very important in the field of architecture modeling, where the level of complexity faced in the modeling does not always allow a prior control. Moreover, the construction of 3D models often passes from the plans, sections and elevations generation in $2 \mathrm{D}$, in which the normals aspect of is certainly not considered in deep.

Returning to the construction phase of 3D geometric models, each construction approach presents its rules and possible problems. Therefore, the possibility to follow some "best practices" during the geometric construction phase may allow a better and more conscious management of the normal problem, optimizing the corrective phase, which requires a considerable man/time hour. In the following paragraphs a geometric analysis will be carried out, in order to highlight some relations between surface generation and normal distribution. Moreover, some corrective approaches will be compared, directly verifying their application on a complex case study, which presents considerable problems on the consistent normal orientation.

\section{GEOMETRIC ANALYIS}

The digital representation of forms proposes more and more advanced procedures and modeling systems that in recent years are shifting the attention towards the relationships between entities (such as generative systems) rather than on their morphology. In this wide and constantly changing framework we can however distinguish two main classes of representation methods: the first aimed at the continuous description of forms by means of mathematical NURBS equations (defined mathematical representation), the second that makes use of lists of data to describe polyhedral, and therefore discrete, meshes (polygonal representation) that approximate spatial forms. 
The first method, using a parametric language, creates forms in continuity with the geometric surface generation methods and ensures the metric control of the entities. For this reason, architecture has in mathematical description its privileged method of representation. During the generation phase of the forms, the differential qualities that characterise lines and surfaces are defined. Among these properties, the directions of the surface normals (which remain unchanged in the subsequent tessellation phase for polygonal environment export) are particularly significant, since they define the faces where it is possible to apply rendering calculation. For this it is useful to investigate the geometric principles underlying the generation of forms in mathematical representation, providing useful and shared criteria in order to control and design with awareness the properties of the represented entities.

The main character of the mathematical representation refers to the description of surfaces as two-dimensional sets of points determined according to the values of two parameters. The description of the entities in parametric language serves to guarantee the mapping between values of the parametric space (domain) and the geometric coordinates, to be able to represent them without ambiguity. The first effect that this type of representation produces is the well-known question of the quadrilateral nature of the patches with which the operator will have to deal often using specific modelling strategies (e.g. trimmed surfaces) to describe any spatial form.

The representation by surfaces is also extended to the representation of solids, which are analysed according to the surfaces that enclose the volume. From a topological point of view this means identifying an inside and an outside. To univocally determine whether a point is inside or outside a volume, the B-rep (boundary representation) technique is used, for which the condition is verified that all surfaces must be adjacent and share an edge.

If this topological analysis is confirmed, the entity is manifold type and the normal vectors are coherently oriented to be turned outwards. The other entities recognized as valid by digital systems, but which cannot be related to the real world, are those composed of several surfaces related to each other without having the property of defining a volume (non-manifold entities which include skins or open polysurfaces). These entities organize the adjacent relations of the single surfaces to uniform the directions of the normal vectors without offering the possibility of predicting their orientation.

Besides, the case of entities composed by single surfaces is possible, where the normal directions depend exclusively on the way the generative curves were created. In addition, all surfaces that cannot be oriented should be mentioned.

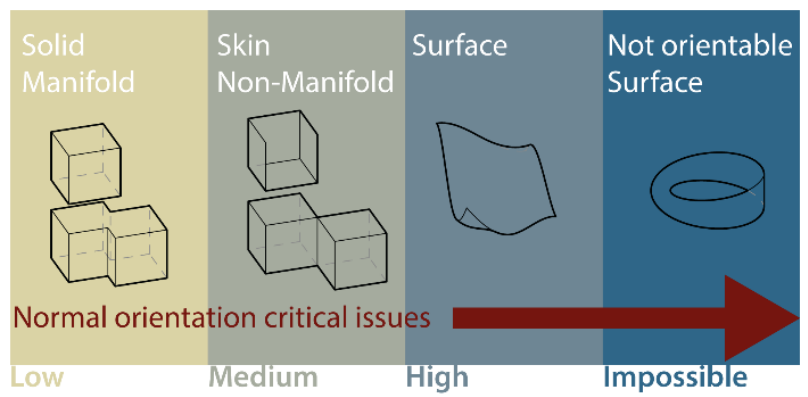

Figure 3: Normal orientation critical issues.
For example, a Moebius strip, which can be formed by twisting a strip of paper one half turn and pasting the two ends together, cannot be oriented. In this case, if normal vectors are drawn on the surface, then the half-twist means that there will be a sudden reversal of the normal directions.

From this first analysis we can therefore distinguish some classes of entities typical of the mathematical representation that present, in increasing order, the problem of normal orientation (Fig. 03): - Manifold entities (closed polysurfaces, i.e. solids),

- Non-manifold and open entities (open polysurfaces or skins),

- Simple surfaces,

- Not orientable surfaces

In general, in architectural representation, the approach to 3D modeling should favour the solid representation of the elements by automatically resolving the normal orientation. Moreover, this procedure would lead to a model consistent with the real nature of the architectural object and would simplify most of the modelling procedures (for example using boolean operations) and finally would be able to provide a digital representation useful to other fields of application such as 3D printing or finite element analysis.

The presence of complex architectural elements, however, makes the use of this approach not always possible, orienting the choices on other modeling strategies. In the case of a surface modeling it is important to control the generative phase of the shapes because, as anticipated, it is the key step for the control of the orientation of surface normals.

The direction of the tangents of a NURBS curve depends on its parameter, which in turn follows the order in which the control points are created in the scene. The direction of tangents of the generating lines will define the direction of the surface normals. As an example, let's imagine in a right-handed coordinate system the creation of a closed polyline that follows a clockwise order and construct a surface whose edge is defined by the polyline itself: the direction of the surface normal will be turned downwards coherently with the right-hand applied to the calculation of the cross product of the vectors defined by the tangent lines.

For this, a brief investigation that analyses from geometric genesis point of view some families of recurrent surfaces in architecture is suggested, evaluating the logic that define the normal directions (Fig. 04). This experimental step on geometrical construction is aimed to understand better the relationship between surface generation approach and normal distribution, in order to be acquainted during modeling step, carrying on an a-priori normal check considered in Figure 02.

In translational surfaces, the normal directions depend not only by the tangent directions of profile and path lines, but also on the role they play in the generation of the surface (or polysurface), and which for this class of surfaces is interchangeable.

Also, in the case of rotational surfaces, the tangents of the profile curve together with the modalities with which it is possible to identify the axis of revolution condition the orientation of the surface normals. In the case of helical surfaces, the direction of surface normals is instead conditioned by the directions of the tangents of the cylindrical helix and the generating line. For surfaces generated by projection, such as the elliptical cone, the normal directions are defined by the tangent directions of the generator curve and the position of the vertex of the cone (if placed in the positive or negative direction of the coordinate system). 


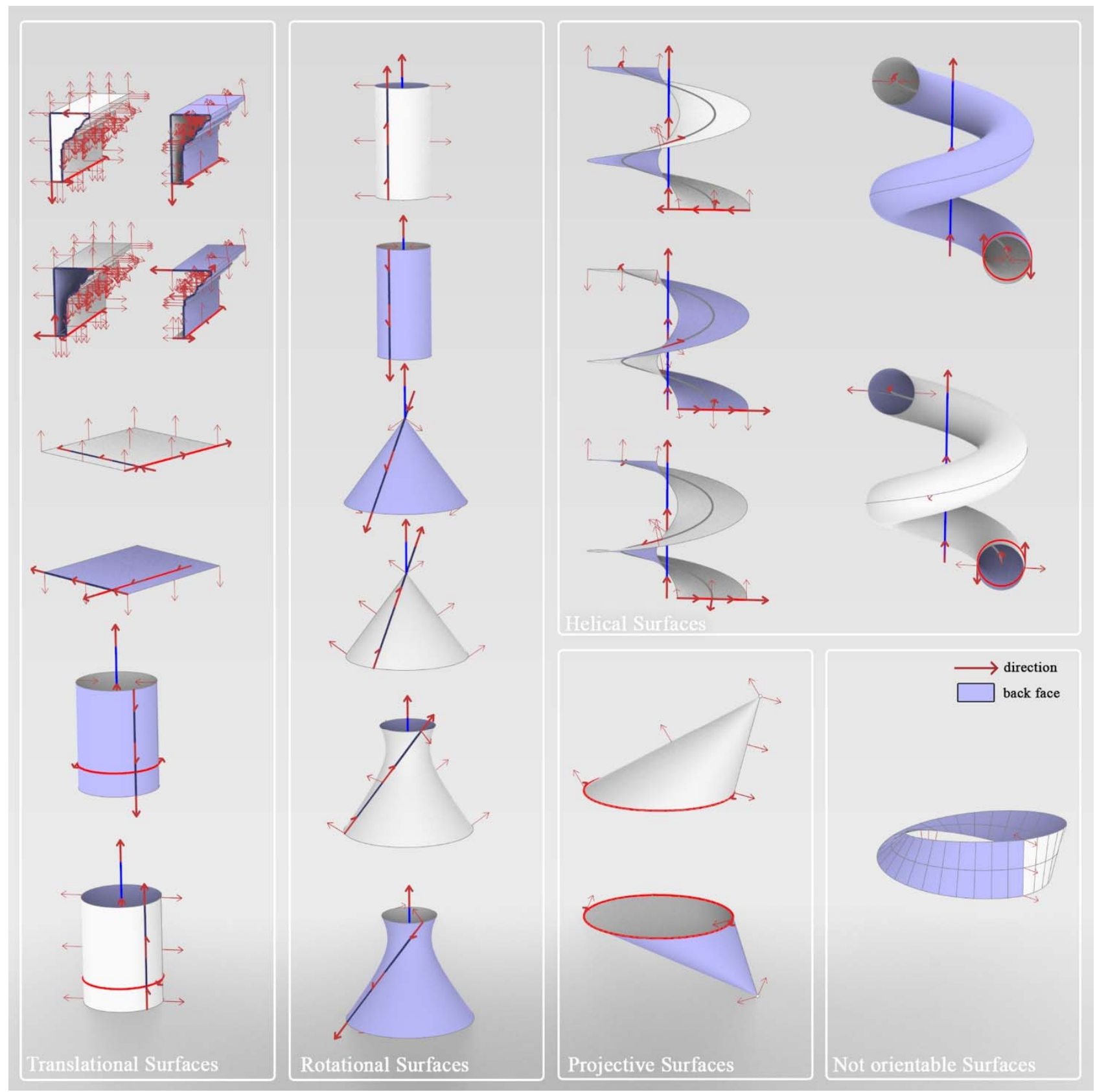

Figure 4: Relationships between geometric surface generation and normal directions.

\section{PARAMETRIC MODEL CORRECTION FOR AR}

In this paragraph we refer to the methods of optimization and correction of parametric models made without keeping in consideration an a priori control of surface normals. The case study analysed regards one of the few preserved Renaissance examples of the Este period, the villa del Verginese (Fig. 5), a two-storey building on which an integrated survey campaign has been yet carried out in order to analyse in deep the structure (Russo, 2018). Starting from integrated range-based and imagebased data, geometrical information has been extracted, creating the whole $3 \mathrm{D}$ reconstructed model of the building, both considering outdoor environment and indoor ambient, with relative vertical connection as a well-preserved Renaissance staircase. During the modeling phase, no attention has been devoted to the normal distribution, an almost frequent condition in architecture modeling. The experimentation about normal correction starts from this modeling step.
Models not subjected to careful previous verification and final correction may present several visualization problems in AR (Fig. 6) due to the inconsistent distribution of model normals (Fig. 7).

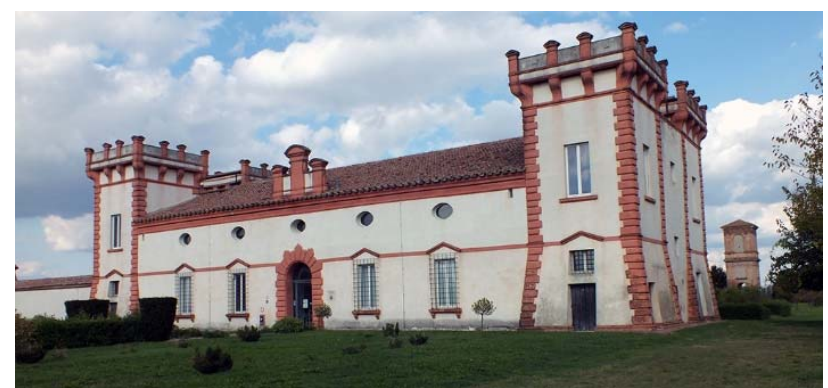

Figure 5: Picture of villa del Verginese (Ferrara) 


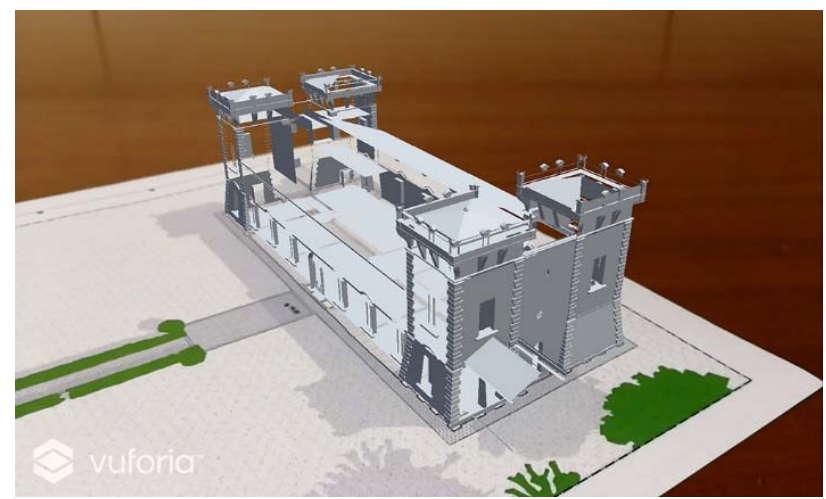

Figure 6: Relationships between geometric surface generation and normal directions.

Unfortunately, most of the modeling tools do not allow the automatic correction of normals, especially when there are geometric defects such as the presence of gaps, unintentionally intersecting primitives and T-junctions. This problem has already been addressed in other areas (Borodin et al. 2004) proposing an algorithm able to consistently re-orient the normals, even in the presence of the mentioned geometrical defects. This tool, in our knowledge, is not yet available in the main modeling software products. Therefore, the only corrective solution accessible to a user who is not expert on algorithms or computer science is to trust in manual or partially-automatic correction, which we will describe later.

AR software operates in a numerical environment, so it is necessary to perform a transformation of the mathematical model known as tessellation (Baglioni, 2008). The present experimentation is carried out on two different fronts: the correction of the mathematical model and the correction of the polygonal model deriving from the tessellation of the mathematical model, verifying which of the two procedures is quicker to follow and allows obtaining the best result.

The workflow is considered associating the most suitable software environment to each processing phase (mathematical environment for geometric modeling, numerical environment for materials, texturing, lighting and rendering), but it can be modified according to the purpose of the augmented model and the personal skills on modeling platforms.

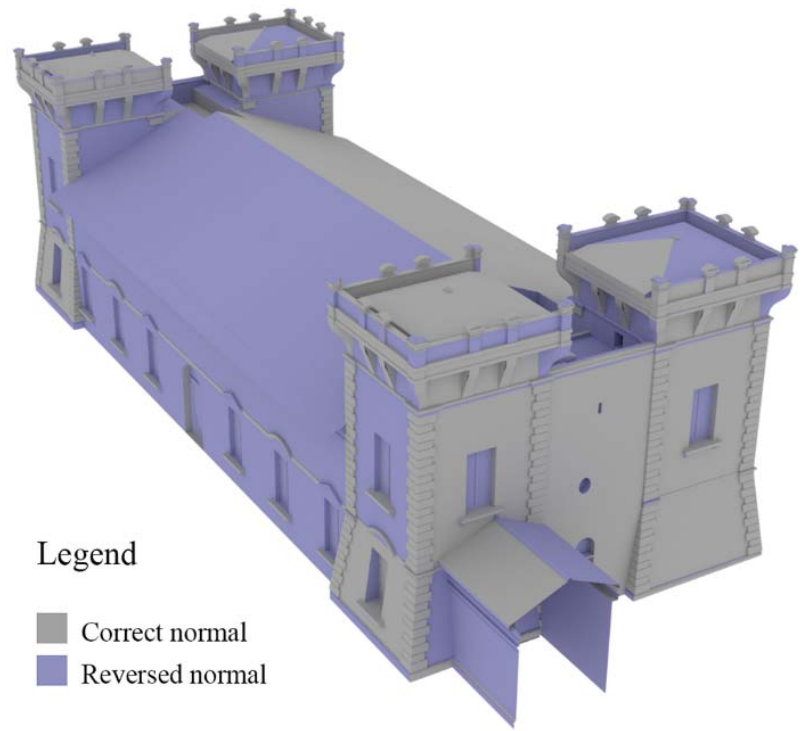

Figure 7: 3D model of Villa del Verginese, highlighting normal surface distribution.
If the modelling project aim is to obtain AR of a monochrome model, because the interest is focused only on the architecture geometry rather than the definition of materials and textures analysis, the passage of material/texture assignment can be omitted. This exclusion will also speed up the task of verifying normals.

The present case study investigates both the procedure: the first one leads to the visualization in AR of monochromatic models of high geometric complexity, through the example of a renaissance staircase inside the Villa del Verginese. The second one is aimed at obtaining the visualization of complex geometric models, characterized also by their chromatic component, such as the $3 \mathrm{D}$ modeling of the exterior of the villa.

Regarding the correction in the mathematical environment, the principle to follow is to join contiguous surfaces since the parametric definition of the two or more joined surfaces becomes consistent, as is the direction of their normals. In this way, the possible reverse direction is faster because it is applied to less elements. The ideal situation would be to reach closed polysurfaces, as this type of geometry automatically involves the correct orientation of the normals. Although this is a desirable situation, it is not always feasible for the geometric complexity that characterizes architecture models and the priority assigned to the semantic classification of the model through distinct layers. The semantic distinction, which reflects the subdivision of the model into architectural elements, or into materials or other, involves a division of the model into reduced and often noncontinuous elements. So, if on the one hand the join of all the geometries in a single layer would benefit (for the normal correction), this procedure is not admissible at the expense of maintaining a structured model according to study and work needs.

The workflow proposed starts with a general cleaning of the model in order to eliminate everything that will not have to be seen in the AR application. In this way the check and correction of normals will be directed to a smaller number of elements, and their decrease will also benefit the visualization in RTR. Subsequently it will be possible to start, by activating one layer at a time, the already mentioned join operations, aiming to obtain as many closed polysurfaces as possible, or in any case the join of several elements into open polysurfaces. The next step is to check the normal orientation and possibly the inversion of their direction. Once the verification has been carried out on all the layers of the model, it will be possible to proceed with the tessellation and the export, taking care to reduce the polygon number to the minimum. The cited workflow was followed in our case study. For the realization of the AR application of the Villa del Verginese, a mathematical model was used, structured through the semantic classification of the several construction materials (white plaster, wood, dark frames, etc.).

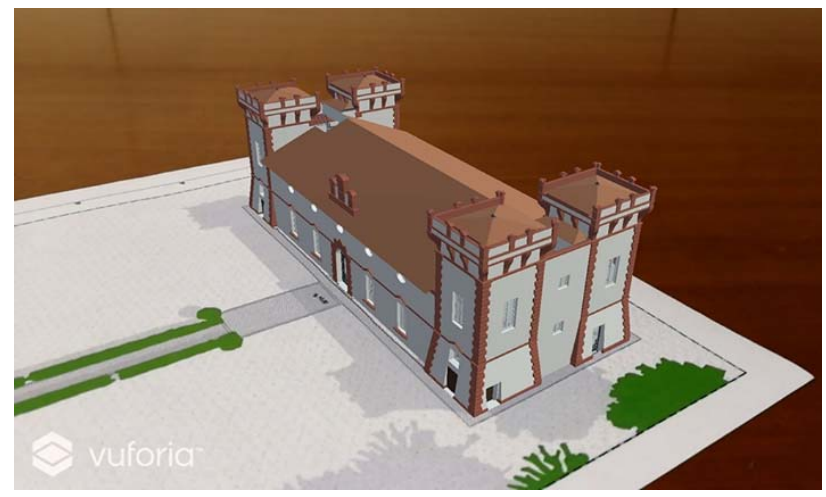

Figure 8: Final AR of Villa del Verginese, developed in Unity with Vuforia plugin. 
This model was checked and corrected, tessellated and exported, and then imported into a numerical software for the material assignment. Finally, the polygonal model was imported into the RTR Unity software and in AR app (Vuforia) for mobile devices, using the plan of the villa as a target for tracking (Fig. 8).

The same workflow was also applied to visualize the model of the Renaissance staircase (Fig. 9). In this case, since it was not necessary to use model structured in different layers because only the architecture geometry was considered interesting (and not the chromatic component), it was decided to merge all the elements into a single layer and create a single closed polysurfaces. This solution has considerably shortened the times for checking and correcting of the normals at about one-third of the working hours.

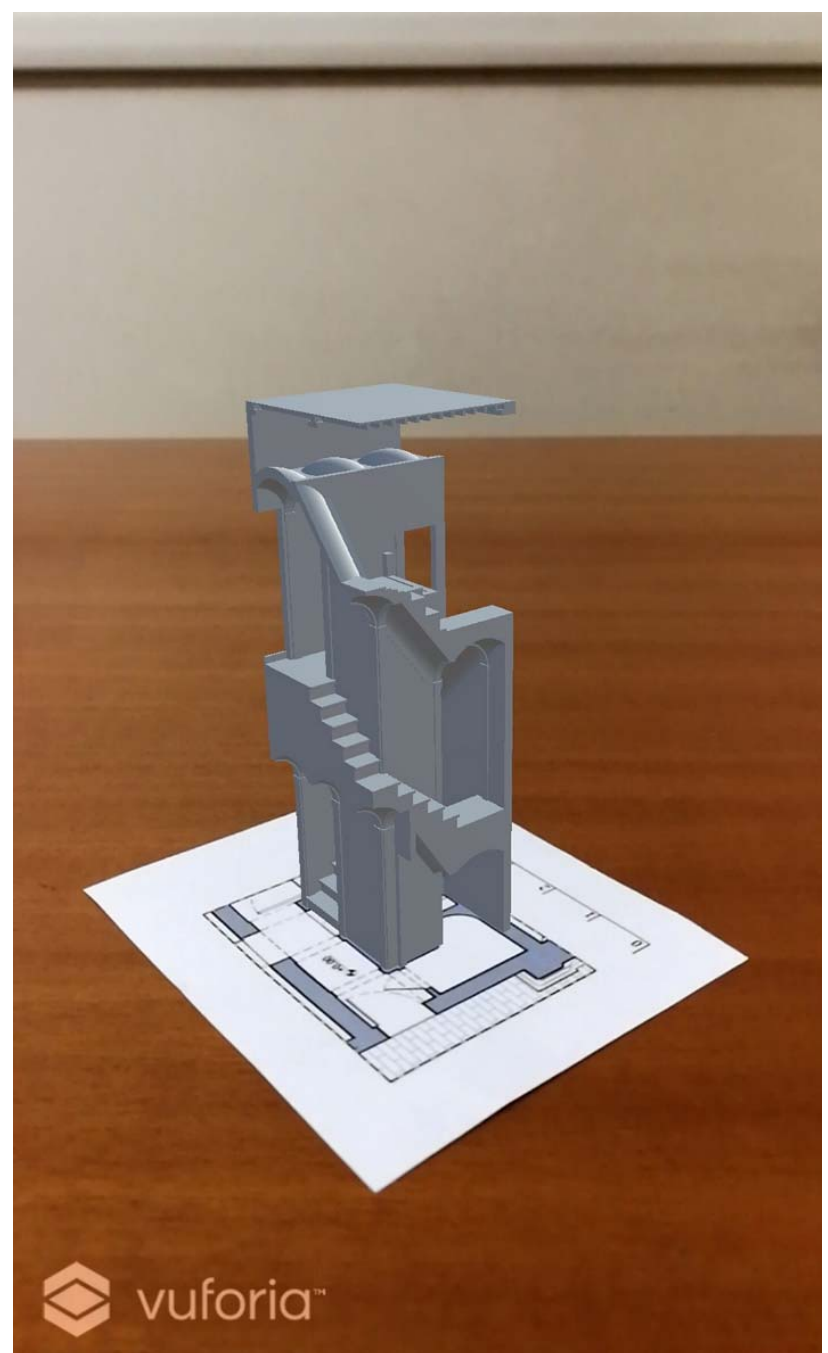

Figure 9: AR of the renaissance staircase, developed in Unity with Vuforia plugin.

The checking and correction of the normals of a parametric model in a polygonal environment is much longer. Polygonal modeling software works with meshes that are defined by points, edges and faces, and not by mathematical parameters. For this reason, the correction is manually applied for each single polygon which presents inverted normal condition. Another solution consists in using automatic algorithms which, however, as already mentioned, can encounter bottlenecks in particularly complex model application. Compared to the correction in the mathematical environment, the higher time spent depends on the impossibility of working on fewer elements, because they are not parametrically joined. It also depends on the higher number of elements obtained after tessellation of the mathematical surfaces.
A different approach concerns the polygonal models generated directly in their environment. In this case the normal analysis happens simultaneously during modeling step, verifying normal coherence distribution and avoiding the final check.

\section{CONCLUSIONS}

Augmented Reality represents a growing communication channel, responding to the need to expand reality with additional information, offering easy and engaging access to digital data. AR for architectural representation allows a simple interaction with $3 \mathrm{D}$ models, facilitating spatial understanding of complex forms and topological relationship between parts. In the last decade different developments in the pipeline process have seen a significant advancement in technological and algorithmic aspects, paying less attention to $3 \mathrm{D}$ modeling generation. The latter aspect is the focus of our contribution.

As shown, the main problem, found in 3D models for AR, is the inconsistent orientation of surface normals, which causes visualization defects in the RTR. The study was carried out by analyzing both the geometric construction phase of the shapes and the possible subsequent correction of "damaged" models.

From the geometrical analysis the variables that help define the orientation of the normal of parametric surfaces are difficult to control during the creation phase. Moreover, architectural modeling often relies on 2D drawings (i.e. plans, sections, elevations), which could contain topological errors (i.e. open edges, overlaps, etc.). These "defects" do not cause problems in the 2D environment and often go unnoticed but result critical in creating topologically correct 3D models. Besides, this bottleneck stresses the deep difference between 2D and 3D approach both from methodological and topological point of view, requiring an a-priori definition of the aim of the $3 \mathrm{D}$ model and the virtual representation platform, which is almost impossible during 2D drawing activity. Starting form this statement, it is possible to establish a priority index for a good modeling approach, which can reduce the problem of normals only to few critical cases. Regarding the normal fixing activity of parametric models, it is considered convenient to perform the correction directly in the mathematical environment because, with the same results, the time dedicated to these operations is lower. This difference has been verified facing different correction processes: the first has been applied on a whole complex building, Villa del Verginese, an ancient Renaissance artefact located near Ferrara city. The second focused on one architectonic element of the villa, the Renaissance staircase.

The results obtained have demonstrated the importance both in the modeling procedure choices and in the geometry quality control, in the meanwhile highlighting a still rigid pipeline demanded to personal modeling skills and perception of the whole AR process. Overlooking the nearly future, a better integration between modeling process and AR creation is desirable, introducing automatic instruments which override bottlenecks, improving Augmented Architecture applications.

\section{REFERENCES}

Arduini, G., 2012. La realtà aumentata e nuove prospettive educative. In: Ripensare La pedagogia, Ri-pensare L'educazione, Education Sciences \& Society, Vol. 2, pp. 209-216

Azuma, R., 1997. A Survey of Augmented Reality. Presence: Teleoperators and Virtual Environments, Vol. 6 (4), pp. 355-385.

Azuma, R., Hoff, B., Neely, H., Sarfaty, R., 1999. A MotionStabilized Outdoor Augmented Reality System. In: Proc. IEEE Virtual Reality, IEEE CS Press, Los Alamitos, Calif., pp. 252259. 
Baglioni, L., 2008. La discretizzazione delle superfici continue. Tesi di dottorato di ricerca in Scienze della Rappresentazione e del Rilievo, 229 p.

Barlozzini, P., Carnevali, L., Lanfranchi, F., Menconero, S., Russo, M., 2018. Analisi di opere non realizzate: rinascita virtuale di una architettura di Pier Luigi Nervi. In: Rappresentazione/Materiale/Immateriale - Drawing as (in)tangible representation $X V$ Congresso UID, Gangemi Editore, Milano, pp. 397-404.

Billinghurst, M., Kato, H., Poupyrev, I., 2001. The Magic-Book - Moving Seamlessly between Reality and Virtuality. IEEE Computer Graphics and Applications, Vol. 21 (3), pp. 2-4.

Bonacini, E., 2011. Nuove tecnologie per la fruizione e la valorizzazione del patrimonio culturale. Aracne, Roma.

Borodin, P., Zachmann, G., Klein, R., 2004. Consistent Normal Orientation for Polygonal Meshes. In: IEEE Proceedings Computer Graphics International, Crete, Greece, pp. 1-8.

Carmigniani, J., Furht, B., Anisetti, M., Ceravolo, P., Damiani, E., Ivkovic, M., 2011. Augmented reality technologies, systems and applications. Multimed Tools Appl, Vol. 51, pp. 341-377.

Choudary, O., Charvillat, V., Grigoras, R., Gurdjos, P., 2009. MARCH: Mobile Augmented Reality for Cultural Heritage. MM'09, Beijing, pp. 1023-1024.

Coorg, S., Teller, S., 1997. Real-time occlusion culling for models with large occluders. Proceeding of the Symposium on Interactive 3D graphics (I3D '97), pp. 83-ff.

De Paolis, L.T., 2012. Applicazione interattiva di realtà aumentata per $\mathrm{i}$ beni culturali. SCIentific RESearch and Information Technology (SCIRES-IT), Vol 2 (1), pp. 121-132.

Eberly, D.H., 2006. 3D Game Engine Design: A Practical Approach to Real-Time Computer Graphics. Morgan Kaufmann Publishers, United States.

Empler, T., 2017. ICT to Communicate, Represent and Enhance an Archeological Area. Putting tradition into Practice: Heritage, Place and Design, Proceedings of 5th INTBAU International Annual Event, Springer, pp. 692-701.

Frontoni, E., Pierdicca, R., Quattrini, R., Clini, P., 2016. Improving the Development of AR Application for Artwork Collections with Standard Data Layer. Augmented Reality, Virtual Reality, and Computer Graphics, pp. 435-443.

Guidi, G., Russo, M., Angheleddu, D., 2014. 3D Survey and virtual reconstruction of archaeological sites. In: Digital Applications in Archaeology and Cultural Heritage, 1(2014), pp. 55-69

Haugstvedt, A.C., Krogstie, J., 2012. Mobile Augmented Reality for Cultural Heritage: A Technology Acceptance Study. IEEE International Symposium on Mixed and Augmented Reality, pp. 247-255.

Luhmann, T., Robson, S., Kyle, S., Boehm, J., 2014. Close Range photogrammetry and $3 D$ imaging, 2nd ed., 684. Berlin, Germany: De Gruyter.

Milgram, P. and Kishino, F., 1994. A Taxonomy of Mixed Reality Visual Displays. IEICE Trans. Information Systems, Vol. E77-D (12), pp. 1321-1329.

Montani, C., Tarini M., Cignoni, P., 2006. Ambient Occlusion and Edge Cueing for Enhancing Real Time Molecular Visualization. IEEE Transactions on Visualization \& Computer Graphics, Vol. 12 (5), pp. 1237-1244.
Mortara, M., Catalano, C.E., Bellotti, F., Fiucci, G., HouryPanchetti, M., Petridis, P. 2014. Learning cultural heritage by serious games. Journal of Cultural Heritage, Vol. 15 (3), pp. 318-325.

Murru, G., Fratarcangeli, M., Empler, T., 2013. Practical Augmented Visualization on Handheld Devices for Cultural Heritage. In: 21th International Conference in Central Europe on Computer Graphics, Visualization and Computer Vision (WSCG), pp. 97-103.

Panciroli, C., Macauda, A., 2018. Heritage education and augmented reality: what prospects. Italian Journal of Educational Research, Vol. IX (20), pp. 48-62.

Potenziani, M., Callieri, M., Dellepiane, M., Corsini, M., Ponchio, F., Scopigno, R., 2015. 3DHOP: 3D heritage online presenter. Journal of Computers \& Graphics, pp. 129-141.

Rabbi, I., Ulla, S., 2013. A Survey on Augmented Reality Challenges and Tracking. Acta Graphica, Vol. 24 (1-2), pp. 29.46 .

Remondino, F., Spera, M.G., Nocerino, E., Menna, F., Nex, F., 2014. State of the art in high density image matching. Photogrammetric Record, 29 (146), pp. 144-66.

Russo, M., 2018. Il Palazzo del Verginese: una Delizia Estense nascosta. Sapienza Università Editrice. Collana Materiali e Documenti, open access, Vol. 30, 222 p.

Sdegno, A., 2018. Augmented Visualization: New Technologies for Communicating Architecture. Computational Morphologies. Design Rules Between Organic Models and Responsive Architecture, Springer, pp. 197-202.

Smith, D., 2016. Augmented Reality in Art Education. The University of Arizona Libraries. http://arizona.openrepository.com/arizona/handle/10150/621860

Sutherland, I., 1968. A Head-Mounted Three-Dimensional Display. In: Fall Joint Computer Conf., Am. Federation of Information Processing Soc. (AFIPS) Conf. Proc., 33, Thompson Books, Washington, D.C., pp. 757-764.

Tom Dieck, M.C., Jung, T., 2018. A theoretical model of mobile augmented reality acceptance in urban heritage tourism. Current Issues in Tourism, Vol. 21 (2), pp. 154-174.

Van Krevelen, D.W.F., Poelman, R., 2010. A Survey of Augmented Reality Technologies, Applications and Limitations. International Journal of Virtual Reality, Vol. 9 (2), pp. 1-20.

Vlahakis, V., Karigiannis, J., Tsotros, M., Gounaris, M., Almeida, L., Stricker, D., Gleue, T., Christou, I.T., Carlucci, R., Ioannidis, N., 2001. ARCHEOGUIDE: First results of an Augmented Reality, Mobile Computing System in Cultural Heritage Sites. In: Proceeding of the 2001 conference on Virtual reality, archaeology, and cultural heritage (VAST), pp. 131-140.

Wu, H. K., Wen-Yu Lee, S., Yi Chang, H., Liang, J-C., 2013. Current status, opportunities and challenges of augmented reality in education. Computers \& Education, Vol. 62, pp. 41-49.

Younes, G., Kahil, R., Jallad, M., Asmara, D., Elhajj, I., Turkiyyah, G., Al-Harithy, H., 2017. Virtual and augmented reality for rich interaction with cultural heritage sites: A case study from the Roman Theater at Byblos. Digital Applications in Archaeology and Cultural Heritage, Vol. 5, pp. 1-9. 